\title{
Extraction and purification of the H9N2 virus nucleoprotein: A simple and practical method
}

\author{
Seyedeh Saeedeh Hosseini ${ }^{1}$, Kayvan Taheri ${ }^{2}$, Seyedeh Marzieh Hosseini ${ }^{3}$, Mehrdad Gholami ${ }^{4}$, \\ Ebrahim Kouhsari*5, Elahe Edalati ${ }^{1}$, Rasoul Madani ${ }^{1}$, Rokhsare Mohammadzadeh ${ }^{5}$, \\ Abed Zahedi Bialvaei ${ }^{5}$, Mohammad Sholeh ${ }^{5}$, Fariba Golchin Far ${ }^{1}$ \\ Received: 30 May 2018 \\ Published: 21 Dec 2018
}

\begin{abstract}
Background: Avian Influenza disease annually entails a significant economic loss to the poultry industry around the world. Influenza virus is a polymorphic virus of the orthomyxoviridae family (single-stranded RNA genome), and nucleoprotein (NP) is the structural and internal protein of the virus. The aim of the work was to purify nucleoprotein for further investigations with a simple, low-cost, fast and practical method.

Methods: In this study, H9N2 influenza virus was isolated in specific pathogen-free embryonated chicken eggs by allantoically inoculating 103 to 105 egg-infective doses (EID50) for 9 to 11 days, purified by $10 \%$ (W/V) polyethylene glycol (PEG) 6000 with a sucrose gradient of $60 \%$ to $30 \%$. The influenza virus proteins were collected and prepared as fractions by preparative electrophoresis. Finally, the purified NP was subjected to sodium dodecyl sulfate polyacrylamide gel electrophoresis (SDS-PAGE) and Western blot procedures.

Results: The protein analysis with SDS-PAGE and silver nitrate staining indicated that the desired samples contained purified nucleoprotein and lacked other viral proteins. The results of the investigation of lyophilized fractions containing nucleoprotein on the SDS-PAGE revealed the absence of viral RNA in nucleoprotein and its high purity.

Conclusion: According to this study, purified nucleoprotein can be used to produce nucleoprotein vaccines, as well as to study structural, molecular and diagnostic and therapeutic materials.
\end{abstract}

Keywords: Avian influenza virus, H9N2, Nucleoprotein, Extraction, Purification

Conflicts of Interest: None declared

Funding: None

*This work has been published under CC BY-NC-SA 1.0 license.

Copyright $₫$ Iran University of Medical Sciences

Cite this article as: Hosseini SS, Taheri K, Hosseini SM, Gholami M, Kouhsari E, Edalati E, Madani R, Mohammadzadeh R, Zahedi Bialvaei A, Sholeh M, Golchin Far F. Extraction and purification of the H9N2 virus nucleoprotein: A simple and practical method. Med J Islam Repub Iran. 2018 (21 Dec);32:128. https://doi.org/10.14196/mjiri.32.128

\section{Introduction}

Avian Influenza is a highly contagious disease that causes symptoms in respiratory, digestive and nervous systems in a wide range of birds and can be transmitted to

Corresponding author: Dr Ebrahim Kouhsari, ekouhsari1987@gmail.com

1. Department of Botany, Biotechnology and Bioinformatics, Payame Noor University, Tehran, Iran

2. Department of Biology, Microbiology, Azad University, Damghan, Iran

3. Department of Biology, Microbiology, Azad University, Karaj, Iran

4. Department of Microbiology and Virology, Faculty of Medicine, Mazandaran University of Medical Sciences, Sari, Iran

5. Department of Microbiology, School of Medicine, Iran University of Medical Sciences, Tehran, Iran mammals, including humans (1). Dramatic changes reassortment between human and avian viruses are due to an-

\section{$\uparrow$ What is "already known" in this topic:}

The nucleoprotein H9N2 influenza virus functions as a significant adaptor molecule between the virus and host cell processes. The purified nucleoprotein is used to detect new virus species caused by interbreeding and their resultant disease and to provide a vaccine for avian influenza for prevention and control programs of influenza in the industry.

\section{$\rightarrow$ What this article adds:}

The aim of this study was to purify virus NP in order to further project with a simple, low-cost, fast and practical method. In the current study, firstly the H9N2 virus identified and the locations of the viral proteins were determined. Subsequently the nucleoprotein was purified with adequate and accurate information about the viral proteins. 
nual epidemics and pandemics (2). To date, the most appropriate method for fighting avian influenza is control and eradication. An effective human influenza vaccine development is a critical component to control and prevent an influenza pandemic (3). Influenza virus is an enveloped, single-stranded negative-sense RNA segmented virus (3). Based on the two proteins on the surface of the virus; hemagglutinin $(\mathrm{H})$, and neuraminidase $(\mathrm{N})$, influenza $\mathrm{A}$ viruses are divided into $15 \mathrm{H}$ and $9 \mathrm{~N}$ subtypes (4). The proteins play key roles in infecting a host cell. In addition, inside the envelope are segmented single-stranded RNA and nucleoprotein (NP) (5). Avian influenza (AI) H9N2 subtype is generally reported as the primary isolate from turkey flocks in Wisconsin in America in 1966, then the virus has been reported and circulated among various countries (6). Although H9N2 is a low-pathogenic virus, the disease has been observed in all types of poultry in the field (7). Because of the wide range of the virus and the great losses it caused, initially a local vaccination program was implemented, but mass vaccination was quickly authorized (8). The virus has spread to other avian species such as chickens (breeders, broilers, and layers) and ostriches (8), it has been panzootic in Eurasia and is now the most dominant subtype of influenza virus in poultry industry (9). The viruses were classified as low pathogenic on the basis of experimental inoculation of chickens by different routes, but under field conditions, they have proved to cause a severe respiratory syndrome in birds, with heavy reductions in egg production and high mortality $(5,7)$. The H9N2 outbreak has been reported in the world from 1944 to 1996 in Europe, Africa, and Asia. Subsequently, in 1997, severe H9N2 cases were reported in many Asian countries (10). All viruses with negative-sense RNA genomes encode an ssRNA binding nucleoprotein (NP) (11). The NP is one of the key determinants of species specificity, RNA encapsulation, transcription, replication, and packaging (11). The NPs have interaction with different molecules such as; PB1, PB2, M1, Importin $\alpha$, F-actin, CRM1/exportin-1 and $\mathrm{BAT}_{1} / \mathrm{UAP}_{56}(11)$. The NP Functions as a key adaptor molecule between the virus and host cell processes $(9,12)$. The purified nucleoprotein is used to detect new virus species caused by interbreeding and their resultant disease and to provide a vaccine for avian influenza for prevention and control programs of influenza in the industry (13). The aim of this study was to purify virus NP in order to further project with a simple, low-cost, fast and practical method.

\section{Methods}

\section{Viral propagation}

In this experimental study, viral propagation was performed by the method described (14). Briefly, the isolates of avian influenza A/Chicken/Iran/259/2014/H9N2 were propagated in specific-pathogen-free chicken embryonated eggs by allantoically inoculating $10^{3}$ to $10^{5}$ egg-infective doses $\left(\mathrm{EID}_{50}\right)$ for 9 to 11 days. Eggs were incubated at $37^{\circ} \mathrm{C}$ with relative humidity of $55 \%$ and were candled daily for 7 days to embryo viability. Allantoic fluid from embryos that died after $24 \mathrm{~h}$ was collected aseptically and tested for hemagglutinating virus by the hemagglutination (HA) test. If no deaths occurred in the eggs after 6 days, all of the eggs were opened aseptically. The allantoic fluids were pooled and inoculated into 5 more embryonated eggs. If no deaths occurred after 7 days in the eggs of the second passage, all the eggs were opened, and the allantoic fluid of each egg was tested for hemagglutinating activity. If deaths occurred in the eggs and the material had a positive HA test, samples were considered positive, and this result was confirmed with the hemagglutinating inhibition (HI) test. Allantoic fluids from embryos showing early mortality after inoculation were tested for bacterial contamination according to routine procedures, and subsequently the infected fluids which contained between $32 \times 10^{5}$ EID 50 of virus per ml were harvested and pooled. Reference stocks were lyophilized and working stocks were stored in ampoules at $-20^{\circ} \mathrm{C}$ (15). However, HA and HI tests were performed by standard methods (15).

\section{Extraction and Purification of nucleoprotein}

The mixture was incubated for 1 hour at $4^{\circ} \mathrm{C}$ and clarified by centrifugation at a speed of $3500 \mathrm{rpm}$ and $4^{\circ} \mathrm{C}$ for 15 minutes. The supernatant was collected, and PEG 5\% (w/v) was added to it. Then, clarified by centrifugation at a speed of $9000 \mathrm{rpm}$ and $4^{\circ} \mathrm{C}$ for 60 minutes, precipitate in TNB buffer was created as a homogenous suspension. Finally, a homogenous suspension was prepared for dialysis to remove PEG from the suspension. The HA test was performed on the sample after dialysis. Then, the dialyzed fluid was ultra-centrifuged at a speed of $20000 \mathrm{rpm}$ and $4^{\circ} \mathrm{C}$ for $2 \mathrm{~h}$. The resulting precipitate was converted into homogeneous suspension in $100 \mathrm{ml}$ of TNE buffer. The HA test was also carried out at this stage. The purification of virus was performed by sucrose density-gradient centrifugation method (16). Finally, the resulting virus samples were lyophilized.

\section{Protein Assay}

For this purpose, Bradford method was performed to determine the protein concentration of the sample according to the previously described method with some modifications (17). In this method, duplicate volumes of bovine serum albumin (BSA) $(10-100 \mathrm{mg} / \mathrm{ml})$ was used to prepare the calibration graph. The basic Laemmli SDS-PAGE procedure was used to separate the protein of interest (18). Afterward, one of the gels was stained with Coomassie blue G-250 (Merck, Germany) and the other gel with silver nitrate. Also, native and functional biological molecules were purified by preparative electrophoresis (model 491 Prep Cell, Bio-Rad, CA), using reduced and non-reduced methods (19). Finally, to determine protein fractions the optical density was read at $280 \mathrm{~nm}$ using a spectrophotometer. The reduced $12 \%$ SDS-PAGE was used to analyze the integrity of the purified influenza virus proteins and the detection of fractions containing nucleoprotein. In brief, $200 \mu \mathrm{l}$ of each protein fraction [with a high absorption] was mixed with 30 $\mu \mathrm{l}$ of 2-mercaptoethanol and incubated for $4 \mathrm{~h}$ at $37^{\circ} \mathrm{C}$. The protein fractions [with high absorption] were dissolved in non-reducing SDS-PAGE loading buffer (without 2-mercaptoethanol) and stained with silver nitrate (20). The protein concentration was assessed with the Bradford method. Finally, in order to confirm H9N2 presence, the purified 
sample was loaded and resolved by SDS-PAGE and proteins were transported to polyvinylidene difluoride (PVDF) membranes. Membrane blocking was done using 5\% bovine serum albumin (BSA), overnight. Primary specific antibody versus H9N2 was used at a 1:1000 dilution, and secondary antibody was used at a 1:10000 dilution (HRP-conjugated). Immunoreactive bands visualized with chemiluminescence HRP substrate (Abcam).

\section{Results}

\section{Protein assay before SDS-PAGE}

A protein assay was performed for influenza virus, and at this stage, BSA was used as a standard in volumes of 100 , 300,500 and $1000 \mathrm{mg} / \mathrm{ml}$, and the results indicated that the volume of the viral solution was appropriate. According to Table 1, the highest and lowest amounts of protein were the sample F act 3 and the sample F act 5 with $387.07 \mathrm{mg} / \mathrm{ml}$ and $212.5 \mathrm{mg} / \mathrm{ml}$, respectively.

\section{SDS-PAGE after protein assay}

Gels were prepared with concentrations of 2.5, 5 and 10 $\mu \mathrm{g}$ of each sample. According to our results (Fig. 1), the molecular weight of the nucleoprotein was estimated between 45 and $66.2 \mathrm{kDa}$.

\section{Preparative electrophoresis results}

The absorption of the fractions was obtained from the purified nucleoprotein at $280 \mathrm{~nm}$ (UV). Graphs 1 and 2 were plotted from the 121 fractions of the reduced and, 123 fractions of the non-reduced methods. Our results showed that 26 fractions from the reduced and 42 fractions from the non-reduced methods had a positive absorption, and these fractions contained viral NP.

\section{SDS-PAGE and Western blotting after protein purifica-} tion

SDS-PAGE was performed for the presence of NP after purification with preparative electrophoresis method (Figs. 2 and 3). According to the molecular weight of NP, fractions 2 and 3 (reduced purification) contained viral NP, but in fractions with non-reduced purification, no band was detected, and no viral protein was isolated. The NP band (H9N2) was verified again by immunoblotting assay (Fig. 4).

\section{Discussion}

The purpose of this work was to prepare and purify the influenza virus nucleoprotein in a simple, low-cost, applied and rapid method. In the current study, firstly the H9N2 virus identified and the locations of the viral proteins were determined. Subsequently the nucleoprotein was purified with adequate and accurate information about the viral proteins. The use of a live virus for propagation is necessary, but epidemiological hygiene needs to be considered, and this issue is more important in influenza viruses due to their genetic rearrangement and transmission potential to humans. However, the advantages of using the inactive virus are due to the lack of infectious potential and long-term maintenance and the absence of contamination with other

\begin{tabular}{ccc}
\multicolumn{3}{c}{ Table 1. Results of Protein assay } \\
\hline Sample name & OD 595nm & Protein $\mathrm{mg} / \mathrm{ml}$ \\
\hline St 100 & 0.08 & 100 \\
St 300 & 0.31 & 300 \\
St 500 & 0.53 & 500 \\
St 1000 & 0.97 & 1000 \\
F act 1 & 0.22 & 212.9 \\
F act 2 & 0.32 & 309.67 \\
F act 3 & 0.40 & 387.07 \\
F act 4 & 0.27 & 261.29 \\
F act 5 & 0.17 & 212.5 \\
\hline
\end{tabular}

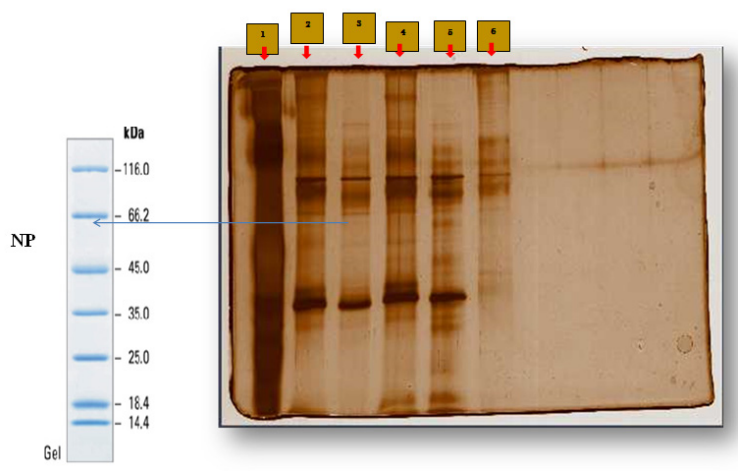

Fig. 1. SDS PAGE gel for absorption (reduction method) protein fractions stained silver nitrate

$1 . F$ act $4(10 \mathrm{mg}), 2 . \mathrm{F}$ act $4(5 \mathrm{mg}), 3 . \mathrm{F}$ act $4(2.5 \mathrm{mg}), 4 . \mathrm{F}$ act $5(10 \mathrm{mg}), 5 . \mathrm{F}$ act $5(5 \mathrm{mg}), 6 . \mathrm{F}$ act $5(2.5 \mathrm{mg})$

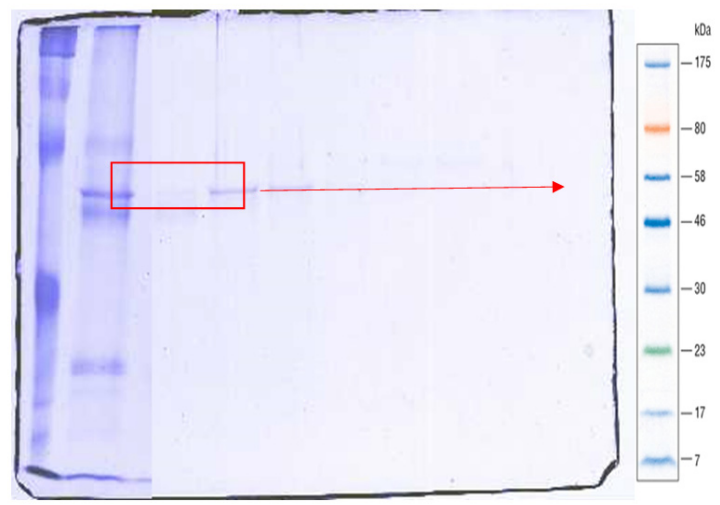

Fig. 2. SDS PAGE gel for adsorption of (reduction method) protein fractions

1. Prestaining Marker, 2. Virus sample before purification, 3. Absorption fraction containing nucleoprotein, 4 . Absorption fraction containing nucleoprotein

microorganisms. The virus from dialysis was lyophilized to facilitate further protein purifications. By SDS-PAGE analyses, the virus protein bands were examined, and it was determined that a nucleoprotein with a molecular weight $\sim 54$ $\mathrm{kDa}$ is clearly identifiable [between bands of 45 to $65 \mathrm{kDa}$ of ladder], and two bands (top and down), which are related to the HA protein, can be identified and separated. However, to ensure that the NP protein can be observed in low amounts of protein and the diluted sample, gels are stained with silver nitrate, which is about 10 to 100 times more accurate than the Coomassie blue. For purity and the presence of the desired protein, we examined the molecular weight and position of the nucleoprotein in terms of movement and detachment in the polyacrylamide gel; samples were run on a preparative electrophoresis machine. The Prep Cell 491 has a special constructional complexity and requires the 


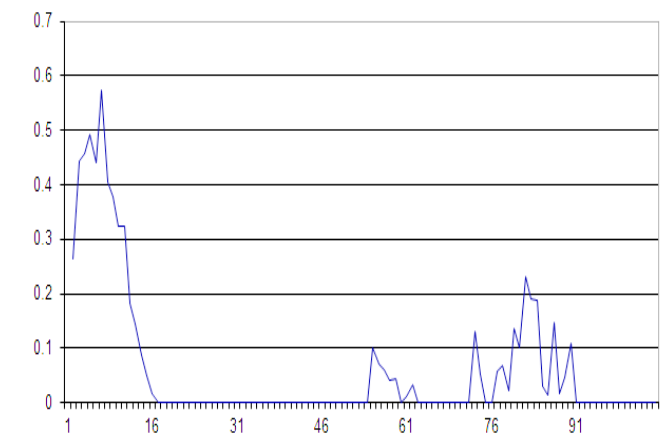

Graph 1. Absorption of 121 fractions from (reduced method) at 280 $\mathrm{nm}$

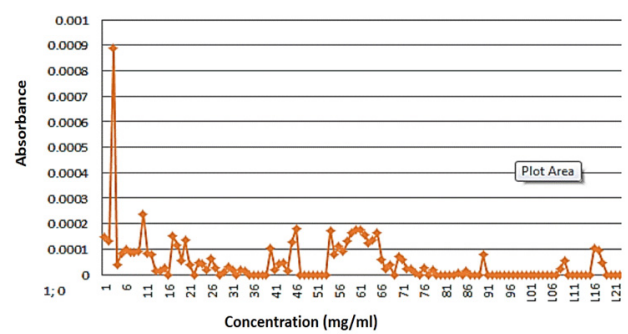

Graph 2. Absorption of 123 fractions from (non-reduced method) at $280 \mathrm{~nm}$

precision and observance of the basic principles of assembling its parts. The components should be tightly assembled and the gel preparation, spraying, fractionation and droplet adjustment steps should be done correctly, and since the application and operation of the device is long, it is necessary to schedule proper testing. The accuracy of the purification of the influenza virus proteins is very important due to the small size and the number of proteins and types of proteins. By adjusting the fractionation device, the volume of $8 \mathrm{ml}$ and duration of 20 minutes for each fraction, this step lasted $48 \mathrm{~h}$. Initially, the fractions contain a protein-free stain which should be discarded. After preparing the fractions and determining their absorbance at $280 \mathrm{~nm}$, the precise and pure presence of the nucleoprotein in fractions 2 and 3 was observed after complete removal of color, and this result was confirmed by SDS-PAGE, but in the non-reducing procedure, no purification was performed, and no band was found in SDS-PAGE. To increase the volume of pure nucleoprotein fractions, fractions containing the nucleoprotein were lyophilized. After lyophilization of the fractions containing the nucleoprotein, protein assay, SDS-PAGE and staining with silver nitrate were performed again. The significant points about the electrophoresis of the influenza virus are the importance of the preparation stages and purification of the virus and the electrophoretic conditions affecting the pattern of electrophoresis of viral proteins. So that with the slightest change, it is possible to observe a change in the electrophoretic pattern of the virus. The lack of similarity of the electrophoretic pattern of various influenza viruses can be attributed to this cause. To ensure the absence of the influenza virus genome in purified nucleoprotein, (because the nucleoprotein and genome of the virus are in the structure of the virus and the nucleoprotein is the viral genome coverage, and together with the RNA, the RNP forms), the resulting samples were electrophoresed.

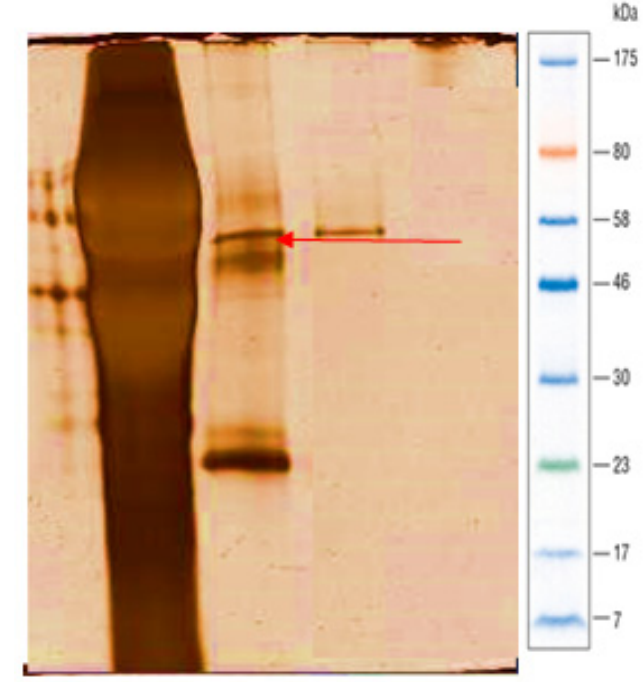

Fig. 3. Gel stained with silver nitrate after lyophilization of fractions (Reduced method)

1. Marker, 2. Viral sample before purification, 3. The sample obtained from lyophilized fractions 2 and 3 (containing nucleoprotein)

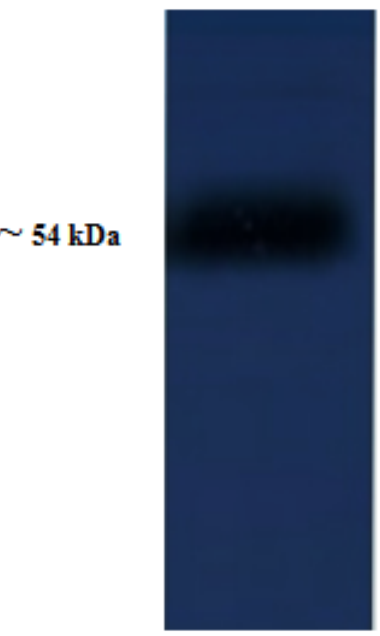

Fig. 4. Identification of purified protein by immunoblotting method

To check the accuracy of the results, the influenza virus before and after lyophilization, and a purified nucleoprotein after lyophilization were tested. The results of electrophoresis indicated no genomic band in the nucleoprotein sample. Thus, during testing, it has been proven that with the help of the Prep Cell 491, we will be able to completely purify the H9N2 avian influenza virus and save time and cost. Purified nucleoprotein can be used to provide nucleoprotein anti-sera against influenza viruses in future researches. Significant points in this study are the high speed and precision of the test results, which can be expressed as a superior advantage over the success of the research. Studies have been conducted on nucleoprotein and different method used for purification of nucleoprotein. Harmon et al., investigated an immunoassay for serological diagnosis of influenza type using a recombinant DNA producing nucleoprotein antigen and monoclonal human antibody IgG (21). They used immunoaffinity chromatography method for NP purification. This method is time-consuming and 
costly, and although the appropriate amount of nucleoprotein was purified, supplementary experiments and a large number of viral samples are required. In the present study, with the least number of the viral samples, nucleoprotein can be obtained. Li et al., (2014), isolated and purified the NP by SDS-PAGE for NP purification and then mass spectrometry was performed (22). In the SDS-PAGE electrophoresis method, viral proteins are isolated but not completely purified, and when cutting from the gel, there is a possibility of error. On the other hand, in this method, the accuracy and skill of the researcher are of great importance, and the cost of mass spectrometry is significant for confirmation of the protein.

\section{Conclusion}

Considering the results of our study, the purified nucleoprotein was obtained very dynamically, with high speed, precision, and purity. Purified nucleoprotein can be used to produce nucleoprotein vaccines, as well as to study structural, molecular and diagnostic and therapeutic materials.

\section{Acknowledgments}

We thank our colleagues from Department of Botany, Biotechnology and Bioinformatics, Payame Noor University who provided insight and expertise that greatly assisted the research.

\section{Conflict of Interests}

The authors declare that they have no competing interests.

\section{References}

1. Lee CW, Saif YM. Avian influenza virus. Comparative immunology, Microbiol Infect Dis. 2009;32(4):301-10.

2. Taubenberger JK, Kash JC. Influenza virus evolution, host adaptation, and pandemic formation. Cell Host Microbe. 2010;7(6):440-51.

3. Sui J, Hwang WC, Perez S, Wei G, Aird D, Chen Lm, et al. Structural and functional bases for broad-spectrum neutralization of avian and human influenza A viruses. Nature Struct Molecul Biol. 2009; 16(3):265.

4. Wang TT, Tan GS, Hai R, Pica N, Ngai L, Ekiert DC, et al. Vaccination with a synthetic peptide from the influenza virus hemagglutinin provides protection against distinct viral subtypes. Proceed National Acad Sci. 2010;107(44):18979-84

5. Neumann G, Noda T, Kawaoka Y. Emergence and pandemic potential of swine-origin H1N1 influenza virus. Nature. 2009;459(7249):931.

6.Zhang Y, Yin Y, Bi Y, Wang S, Xu S, Wang J, et al. Molecular and antigenic characterization of $\mathrm{H} 9 \mathrm{~N} 2$ avian influenza virus isolates from chicken flocks between 1998 and 2007 in China. Veterin Microbiol. 2012;156(3-4):285-93.

7. Sun Y, Liu J. H9N2 influenza virus in China: a cause of concern. Protein Cell. 2015;6(1):18-25.

8. Swayne DE. Avian influenza: John Wiley \& Sons; 2009.

9. Majidzadeh AK, Soleimani M, Karimi V, Shojaee-Estabragh A, Fanni A, Mandegar O, et al. Nucleoprotein (NP) Gene Based Phylogenetic Analysis of Iranian H9N2 Avian Influenza Isolates during 1998-2011. Iran J Virol. 2012;6(1):36-42.

10. Su S, Bi Y, Wong G, Gray GC, Gao GF, Li S. Epidemiology, evolution, and recent outbreaks of avian influenza virus in China. Jo Virol. 2015;89(17):8671-6.

11. Kao RY, Yang D, Lau L-S, Tsui WH, Hu L, Dai J, et al. Identification of influenza A nucleoprotein as an antiviral target. Nature Biotechnol. 2010;28(6):600.

12. Portela An, Digard P. The influenza virus nucleoprotein: a multifunctional RNA-binding protein pivotal to virus replication. J General Virol. 2002;83(4):723-34
13. Pica N, Palese P. Toward a universal influenza virus vaccine: prospects and challenges. Ann Rev Med. 2013;64:189-202.

14. Tang S, Li Y, Xia H, Huang J, Zhang Z, Zhu N, et al. Improved methods for isolation of avian influenza virus. J Virolog Methods. 2014;210:22-5.

15. Kawaoka Y, Neumann G. Influenza virus: methods and protocols: Springer; 2012

16. Raschke S, Guan J, Iliakis G. Application of Alkaline Sucrose Gradient Centrifugation in the Analysis of DNA Replication After DNA Damage. DNA Replication: Methods and Protocols. 2009:329-42.

17. Kruger NJ. The Bradford method for protein quantitation. The protein protocols handbook. 2009:17-24.

18. Laemmli UK. Cleavage of structural proteins during the assembly of the head of bacteriophage T4. Nature. 1970;227(5259):680-5.

19. Saraswat M, Musante L, Ravidá A, Shortt B, Byrne B, Holthofer H. Preparative purification of recombinant proteins: current status and future trends. BioMed Res Int. 2013;2013.

20. Zhang P, Tang Y, Liu X, Liu W, Zhang X, Liu H, et al. A novel genotype H9N2 influenza virus possessing human H5N1 internal genomes has been circulating in poultry in eastern China since 1998. J Virol. 2009;83(17):8428-38.

21. Harmon MW, Rota PA, Walls HH, Kendal AP. Antibody response in humans to influenza virus type B host-cell-derived variants after vaccination with standard (egg-derived) vaccine or natural infection. J Clin Microbiol. 1988;26(2):333-7.

22. Li Z, Sun W, Wu D, Gao X, Sun N, Liu N. Mass spectrometry analysis coupled with de novo sequencing reveals amino acid substitutions in nucleocapsid protein from influenza A virus. Int $\mathrm{J}$ Molecul Sci 2014;15(2):2465-74 